\title{
Temperature Dependence of the Switching Field in Nanocrystalline FeNiMoB Microwires
}

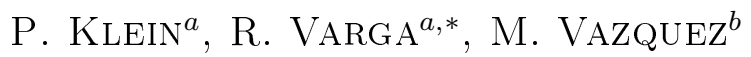 \\ ${ }^{a}$ Institute of Physics, Faculty of Sciences, P. J. Safarik University, Park Angelinum 9, 04154 Košice, Slovakia \\ ${ }^{b}$ Institute of Material Science of Madrid, CSIC, 28049 Madrid, Spain
}

We have studied temperature dependencies of the switching field in as-cast and nanocrystalline glass-coated $\mathrm{Fe}_{40} \mathrm{Ni}_{38} \mathrm{Mo}_{4} \mathrm{~B}_{18}$ microwires. The switching field shows complex temperature dependence in the as-cast state reflecting the complex stress distribution induced during annealing. The temperature dependence of the switching field depends strongly on the stage of nanocrystallization being negative for low temperatures of annealing and positive for annealing at $700 \mathrm{~K}$.

DOI: 10.12693 /APhysPolA.126.64

PACS: $75.50 . \mathrm{Kj}, 75.60 . \mathrm{Ej}, 75.60 . \mathrm{Jk}$

\section{Introduction}

Glass-coated microwires with positive magnetostriction are characterized by a magnetic bistability due to their magnetization process that runs through the single large Barkhausen jump when the external field exceeds the so-called switching field $[1,2]$. Such bistability can be used in many applications like magnetic coding, sensors of magnetic field, mechanical stress, etc. However, the crucial parameter for their application is the time and temperature stability. The solution can be found in nanocrystalline microwires prepared by the heat treatment from amorphous precursors which exhibit much higher stability and very good soft magnetic properties [3]. The temperature dependence of the magnetization process in glass-coated nanocrystalline microwires has been studied by many authors $[4,5]$.

The aim of this paper is to investigate the temperature dependence of the switching field in nanocrystalline microwires based on $\mathrm{Fe}_{40} \mathrm{Ni}_{38} \mathrm{Mo}_{4} \mathrm{~B}_{18}$ composition in order to employ them as temperature sensor.

\section{Experimental}

The study has been performed on glass-coated microwires with nominal composition of $\mathrm{Fe}_{40} \mathrm{Ni}_{38} \mathrm{Mo}_{4} \mathrm{~B}_{18}$ prepared by the Taylor-Ulitovsky method. Diameter of the metal core was $8 \mu \mathrm{m}$ and total diameter was $12 \mu \mathrm{m}$.

The samples were annealed for 1 hour at three different temperatures: $650 \mathrm{~K}, 675 \mathrm{~K}$ and $700 \mathrm{~K}$ (in order to obtain various stage of crystallization) in protective argon atmosphere and finally slowly cooled down.

The switching field has been measured by induction method using triangular waveform to feed primary coil at frequencies $50 \mathrm{~Hz}$ and $500 \mathrm{~Hz}$. The maximum amplitude

\footnotetext{
*corresponding author; e-mail: rvarga@upjs.sk
}

of exciting magnetic field was kept constant $(2400 \mathrm{~A} / \mathrm{m})$ for all measurements. Temperature dependence of the switching field was measured in the temperature range from $80 \mathrm{~K}$ up to $425 \mathrm{~K}$. The length of all samples used in measurement of temperature dependence of the switching field was $10 \mathrm{~cm}$.
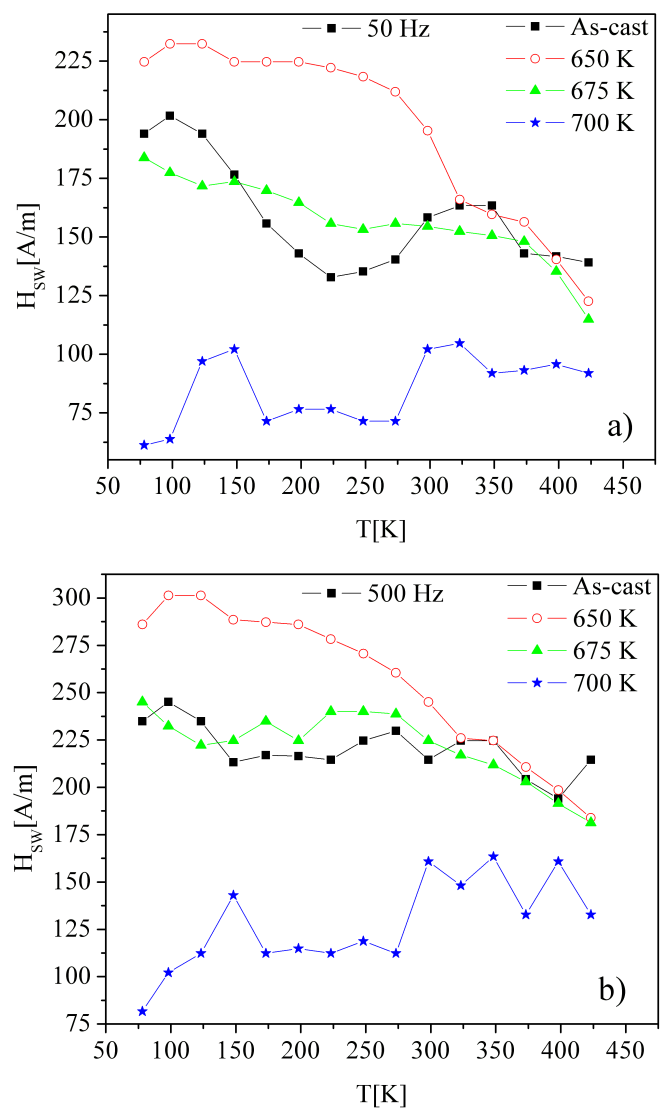

Fig. 1. Temperature dependence of the switching field for amorphous and nanocrystalline FeNiMoB microwires measured at frequency a) $50 \mathrm{~Hz}$ and b) $500 \mathrm{~Hz}$. Temperature of annealing as a parameter. 


\section{Results and discussions}

The drawing and rapid quenching applied during production of microwires results in a strong and complex stress distribution applied on metallic nucleus. Moreover, additional stresses are applied on nucleus due to different thermal expansion coefficients of metallic nucleus and glass-coating of amorphous microwires. As a result, complex temperature distribution is obtained in as-cast amorphous FeNiMoB microwire (see Fig. 1a). It shows two maxima at 100 and $350 \mathrm{~K}$ connected by an enhanced minimum at $220 \mathrm{~K}$. The switching field in bistable microwires consists of two different contributions. Structural relaxation contribution that arises from the mestastable amorphous structure and magnetoelastic contribution that arises from interaction of strong and complex stress distribution with magnetic moments in metallic nucleus. It has been shown that structural relaxation is strongest at low frequencies, whereas magnetoelastic contribution dominates at the frequencies above $200 \mathrm{~Hz}$ [6]. Hence, comparing the temperature dependence of the switching field at low (Fig. 1a) and high (Fig. 1b) frequencies, one can reach the conclusions that strong temperature and stress temperature dependence of structural contribution is responsible for complex temperature dependence of as-cast microwire (complexicity disappears at $500 \mathrm{~Hz}$ where magnetoelastic contribution dominates).

Such a conclusion is supported by measurement of the temperature dependence of switching field in microwires annealed at $650 \mathrm{~K}$. After annealing, the metallic nucleus is in early crystallization stage - the $\gamma$-FeNi crystals are small and well separated [3], which results in the increase of the switching field amplitude. However, the amorphous phase is well relaxed and the structure is homogeneized. Hence, the switching field monotonously decreases with temperature as a result of the variation of stress applied by glass-coating (Fig. 1a and 1b). Moreover, structural relaxation is still present [7] and results in an enhanced decrease of the switching field measured at $50 \mathrm{~Hz}$ above $280 \mathrm{~K}$ (Fig. 1a).

Annealing at $675 \mathrm{~K}$ leads to the optimum nanocrystalline state of metallic nucleus [3] with lower magnetostriction [8]. This results in the decrease of the switching field amplitude to the value of the as-cast microwire. However, its temperature dependence is more continuous and shows weak monotonous decrease with temperature (Fig. 1a and 1b).

Annealing at $700 \mathrm{~K}$ leads to the steep decrease of magnetostriction [8] that results in a decrease of the switching field. In contrary to the previous states, the switching field increases with temperature (Fig. 1a and 1b). The temperature dependence of the switching field is mainly driven by the magnetoelastic contribution. As a result of complex stress distribution variation, the temperature dependence at higher frequencies (Fig. 1b - where the magnetoelastic contribution prevails) shows more complex temperature dependence, comparing to that of measured at $50 \mathrm{~Hz}$, where the structural relaxation contribution should also be taken into account.

\section{Conclusions}

We have studied the temperature dependence of the switching field for the nanocrystalline glass-coated FeNiMoB microwires in different crystallization stage.

The temperature dependence of the switching field depends strongly on the complex stress distribution introduced during production as well as by different thermal expansion coefficient of metallic nucleus and glasscoating. This results in a very complex temperature dependence of the switching field. After annealing, the temperature dependence of the switching field depends on crystallization stage, being negative for annealing bellow the $700 \mathrm{~K}$. Annealing at $700 \mathrm{~K}$, leads to a steep decrease of magnetostriction that results in the low switching field and its positive temperature dependence.

\section{Acknowledgments}

This work was supported by the project NanoCEXmat No. ITMS 26220120019, Slovak VEGA grant. No. 1/0060/13, APVV-0027-11 and APVV-0266-10.

\section{References}

[1] M. Vázquez, Advanced Magnetic Microwires in: Handbook of Magnetism and Advanced Magnetic Materials, Eds. By H. Kronmüller, S. Parkin, John Wiley \& Sons, Chichester, UK 2007, p. 2193.

[2] H. Chiriac, T.A. Ovari, Prog. Mater. Sci. 40, 333 (1996).

[3] E. Komova, M. Varga, R. Varga, P. Vojtanik, J. Bednarcik, J. Kovac, M. Provencio, M. Vazquez, Appl. Phys. Lett. 93, 062502 (2008).

[4] H. Chirac, M. Tibu, T.-A. Óvari, IEEE Trans. Magn. 45, 4286 (2009).

[5] M. Vázquez, A.P. Zhukov, K.L. Garcia, K.R. Pirota, A. Ruiz, J.L. Martinez, M. Knobel, Mater. Sci. Eng. A 375-377, 1145 (2004).

[6] P. Klein, R. Varga, G.A. Badini-Confalonieri, M. Vazquez, IEEE Trans. Magn. 46, 357 (2010).

[7] E. Komova, R. Varga, M. Vazquez, Acta Phys. Pol. 118, 768 (2010).

[8] R. Andrejco, R. Varga, P. Marko, P. Vojtanik, Czech. J. Phys. 52, A113 (2002). 